\title{
Graft Failure Following Allogeneic Stem Cell Transplantation for Primary Myelofibrosis: A Case Report
}

\author{
Max Mendez ${ }^{\mathrm{a}, \mathrm{c}}$, Willem Bujan ${ }^{\mathrm{b}}$, Gabriela Granados ${ }^{\mathrm{a}}$
}

\begin{abstract}
Myelofibrosis is a Philadelphia chromosome-negative myeloproliferative neoplasm characterized by marrow fibrosis, splenomegaly with cytopenias, constitutional symptoms and poor quality of life. Rates of graft failure following allogeneic stem cell transplantation range from 0 to $5 \%$, being more frequent in non-HLA identical donors. Here we present an infrequent complication, a graft failure in a patient following identical sibling donor stem cell transplantation for myelofibrosis with objective indicators of disease remission.
\end{abstract}

Keywords: Primary myelofibrosis; Myeloproliferative neoplasm; Stem cell transplantation; Graft failure; Graft versus host disease

\section{Introduction}

Primary myelofibrosis (PMF) is a Philadelphia chromosomenegative myeloproliferative disorder characterized by bone marrow fibrosis, splenomegaly and constitutional symptoms [1]. It was first described by Gustav Heuck in 1879 [2], and it is also known as agnogenic myeloid metaplasia, chronic idiopathic myelofibrosis and myelofibrosis with myeloid metaplasia [3]. PMF is the least common of all chronic myeloproliferative diseases, with an incidence of 0.2 to 1.5 per

\footnotetext{
Manuscript accepted for publication December 18, 2013

${ }^{\mathrm{a}}$ Haematology Department, Calderon Guardia Hospital, CCSS, 17th Street, San Jose, Costa Rica

${ }^{\mathrm{b}}$ Haematology Department, Hospital Mexico, CCSS, 76th Street, 41st Avenue, La Uruca, San Jose, Costa Rica

${ }^{c}$ Corresponding author: Max Mendez, Haematology Department, Calderon Guardia Hospital, CCSS, 17th Street, San Jose, Costa Rica. Email: maxmendez@runbox.com
}

doi: http://dx.doi.org/10.14740/jmc1639w
100,000 per year $[4,5]$. The median age at presentation is 65 years and $20 \%$ of patients are younger than 55 years at the moment of diagnosis. Current diagnosis of PMF is based on the 2008 World Health Organization criteria [5] and the most consistent clinical findings are splenomegaly, present in up to $90 \%$ of patients, and fatigue in up to $70 \%$ of patients [6]. Two processes have been implicated in the generation of this disease [7]. A first monoclonal proliferation, resulting in the expansion of one or more myeloid lineages with varying degrees of phenotypic differentiation and accumulating cells in spleen [8], and a second step characterized by a reactive polyclonal response in the bone marrow, leading to fibroblast deposition, collagen proliferation, reticulin fibrosis and ineffective hematopoiesis. Clonality is documented in up to $50 \%$ of patients with the analysis of activation mutation in Janus kinase 2 gene (JAK2 V617F) and there is a possible role of platelet derived growth factor (PDGF) and transforming growth factor (TGF) beta in fibrosis development [9].

PMF has a median survival ranging from 2.5 to 5 years. Conventional medical treatment is unsatisfactory and, with the exception of transfusion therapy, none have been shown to confer a survival benefit [10]. To date, myeloablative allogeneic hematopoietic stem cell transplantation (allo-SCT) remains the only modality with a curative potential for PMF, but has been limited for patients under the age of 60 years and for those with HLA-identical sibling donor because of high transplant-related mortality (TRM) $[10,11]$. In myeloablative allo-SCT, Guardiola and colleagues [12] reported one graft failure and 1-year TRM of $27 \%$, with a 3 -year overall survival of $77 \%$ in 55 patients. We present a patient with graft failure in the following matched sibling donor allo-SCT for myelofibrosis and discuss its potential causes.

\section{Case Report}

A32 years old female mother of one child underwent curettage for an incomplete abortion. During the postoperative routine screening, an ultrasound showed grade III splenomegaly and she was referred to the hematologist for consultation. At that moment, she had pancytopenia and the bone marrow biopsy showed extensive grade IV reticulin fibrosis. PMF diagnosis 
was made and she was started on hydroxiurea. Due to the lack of response according to standard criteria [13], she was proposed to consider allo-SCT as a therapeutic option, which she agreed. An HLA-identical sibling donor was available and transplantation was performed under myeloablative conditioning with busulfan and cyclophosphamide. A total of $4.26 \times 10^{6} \mathrm{CD} 34$ positive cells $/ \mathrm{kg}$ were collected from donor and infused without any complication. She received standard prophylactic medications (for example acyclovir, fluconazole, granulocyte-colony stimulating factor (G-CSF), albendazole, sulfametoxazol and metronidazole). graft versus host disease (GvHD) prophylaxis included mofetil micophenolate and cyclosporine. She developed staphylococcus aureus bacteremia the first week after infusion and infection completely resolved with ceftazidime and amikacine. She was discharged from the hospital at day +21 with hemoglobin in $8.1 \mathrm{~g} / \mathrm{dL}, 5 \times 10^{9} / \mathrm{L}$ platelets and $1.5 \times 10^{9} / \mathrm{L}$ leucocytes under supportive G-CSF and followed ambulatory care with medical consults twice weekly. On day 32 postransplantion, hemoglobin level fell to $5.4 \mathrm{~g} / \mathrm{dL}$ and leucocytes maintained in $1.3 \times 10^{9} / \mathrm{L}$. Platelets were less than $2 \times 10^{9} / \mathrm{L}$. Menorrhagia was an evident cause of anemia and was more intense due to thrombocytopenias. Contraceptives were prescribed. Other causes of anemia were discarded and platelets were considered to have a delayed engraftment at that moment. She was on standard prophylactic antibiotic treatment and immunosuppression and the medications were being adjusted according to laboratory results to maintain therapeutic levels. During this period, she received transfusion therapy with packed red blood cells and platelets until bleeding stopped and cytopenias were unresponsive to supportive therapy. Graft failure was suspected and a short course of G-CSF was started with leukocyte recovery from $1 \times 10^{9} / \mathrm{L}$ to $3.2 \times 10^{9} / \mathrm{L}$, but sustained for only few days. Epstein Barr virus antigenemia, citomegalovirus DNA were negative. Assessment of parvovirus B19 and herpes virus 6 were not performed due to the lack of the appropriate molecular techniques. Peripheral blood films showed a few teardrop cells, and a hypocellular marrow aspirate was taken without hemophagocytosis characteristics. A short trial of erythropoietin resulted in small benefits. Abdominal computer tomography scans reported splenic infarcts with rapid involution of the splenomegaly. JAK-2 mutational analysis was negative and single tandem repeats (STR) genotyping showed a complete chimeras. Although she persisted with cytopenias, all other evidence was against graft failure and for that reason donorlymphocyte infusion was not performed. As outpatient, she never developed fever, and C-reactive protein, galactomanan antigen and procalcitonin were all negatives. Bone marrow biopsy taken at day +32 revealed complete absence of fibrosis with a non-cellular marrow and a small paratrabecular area of erythroid production consistent with marrow failure. She continued on medical control and transfusion therapy and at day +73 post stem cell transplant, she presented with jaundice, total bilirrubin in $8.1 \mathrm{mg} / \mathrm{dL}$, GGT in $423 \mathrm{IU} / \mathrm{L}$, alkaline phosphate in $773 \mathrm{IU} / \mathrm{L}$ and elevated transaminases. At this moment, she had extensive skin pruritic rash, with erythematous macules and papules involving $50 \%$ of body surface and watery diarrhoea. Grade IV acute GvHD was diagnosed and confirmed by skin biopsy. She was admitted to the hospital, and intravenous steroids were started for 3 days and then tapered orally. She developed fever, and cultures were taken from blood, urine, stool and sputum. Eventually, all returned negative and there was no response to broadspectrum empiric antibiotics, with persistent fever even after a complete antifungal treatment course. During this time upper gastric intestinal hemorrhage was complicated by persistent thrombocytopenia requiring multiple platelet transfusions. Epsilon-aminocaproic acid was used as a final effort to reduce bleeding without success. On day +89 , she presented a massive gastric hemorrhage, and endoscopic argon plasma coagulation with adrenaline as well as pharmacologic measures were insufficient to save her life.

\section{Discussion}

This patient exemplifies the difficulties in the management of allo-SCT patients. Fungal and viral agents are among the causes of slow recovery of hematopoiesis, but diagnosis is complicated taking into consideration the immunosuppressive therapy and antibiotics prescribed, therefore, a high degree of suspicion is needed by the transplant team in order to carry out the necessary tests to examine the cause of cytopenia in the post-transplant period. Pancytopenia following bone marrow transplantation is a critical complication and must be carefully and extensively evaluated. Rejection, graft failure, infections and medications are among the causes to be considered. An approach to document engraftment in the post-transplant patient is testing for quimerism, analyzing STR by real-time PCR. STRs are patterns of 5 to 50 base pairs repeats found in the intronic region and are sequences used to assess quimerism with very high degree of accuracy. Complete quimerism is $100 \%$ of donor DNA and is associated with acute GvHD. On the other hand, mixed quimerism is a dynamic measure and should be closely monitored, as increasing values are indication of immunotherapy with donor lymphocyte infusion. Graft failure is another cause of pancytopenia post-transplantation. It is defined as a failure to achieve neutrophil count of at least $0.5 \times 10^{9} / \mathrm{L}$ for three consecutive days by day +35 and a platelet count of $20 \times$ $10^{9} / \mathrm{L}$. In our patient, there was neither self-hematopoyesis nor evidence of engraftment by day +40 . The presence of complete chimera confirmed that all circulating cells were donor originated and therefore the difficulty in making a definite diagnosis.

The incidence of graft failure in myeloablative allo-SCT has been documented to be up to $5 \%$ with the majority of 
patients receiving stem cells from non-identical HLA donors $[10,13,14]$. A recently published study of 51 patients with 33 having HLA-identical sibling donor and 27 under myeloablative regimen, showed no graft failures [14]. Deeg and co-workers [15], in 56 patients undergoing allo-SCT documented zero graft failures in the HLA-identical transplants, with three failures in the non-identical HLA group, two of which were unrelated donors. These data confirm that this serious complication is far more frequent in non-identical HLA transplants with the highest incidence in unrelated donor transplants, which was not this case.

\section{Conclusion}

We present a case of graft failure after allogeneic hematopoietic stem cell transplantation for myelofibrosis manifested as pancytopenia in a patient with rapid reduction in spleen size, no residual marrow fibrosis and in remission. One interesting finding was the lack of engraftment even with the presence of a complete chimera and the development of GvHD suggests that donor stem cells might be circulating and were incapable of repopulating the bone marrow due to serious damage in the microenvironment $[16,17]$.

We ruled out some of the most frequent causes of graft failure; however, because of lack of resources, we cannot assure whether or not herpes virus 6 or parvovirus B19 contributed to pancytopenia. Although infrequent in matched sibling donor transplants, graft failure must be considered in all patients with cytopenias following stem cell transplantation and this case provides an example of the difficulties in diagnosis and treatment, and the urge to treat early to avoid further complications.

In developing countries, there is an increased need to improve diagnostic methods and therapeutic options and guidelines should address different strategies of treatment of patients in more resource-limited institutions.

\section{Conflict of Interests}

The authors declare that they have no conflict of interests.

\section{Abbreviations}

PMF: primary myelofibrosis; JAK2/STAT5: Janus kinase/ signal transducers and activators of transcription; PDGF: platelet derived growth factor; TGF: transforming growth factor; HLA: human leukocyte antigen; GvHD: graft versus host disease; $\mathrm{Hb}$ : hemoglobin; WBC: white blood cell; EBV: Epstein Barr virus; CMV: citomegalovirus; GGT: gamma glutamyl transferase; STR: short tandem repeat; Allo-SCT: allogeneic stem cell transplantation; PCR: polymerase chain reaction

\section{References}

1. Barosi G. Myelofibrosis with myeloid metaplasia: diagnostic definition and prognostic classification for clinical studies and treatment guidelines. J Clin Oncol. 1999;17(9):2954-2970.

2. Mesa R, Tefferi A. Approach to Chronic Idiopathic Myelofibrosis (With Extramedullary Hematopoiesis). In Clinical Malignant Hematology. Edited by Sekeres M, Kalaycio M, Bolwell B. McGraw-Hill Professional. 2007;475-486.

3. Mesa RA, Verstovsek S, Cervantes F, Barosi G, Reilly JT, Dupriez B, Levine R, et al. Primary myelofibrosis (PMF), post polycythemia vera myelofibrosis (post-PV MF), post essential thrombocythemia myelofibrosis (post-ET MF), blast phase PMF (PMF-BP): Consensus on terminology by the international working group for myelofibrosis research and treatment (IWG-MRT). Leuk Res. 2007;31(6):737-740.

4. Mesa RA, Silverstein MN, Jacobsen SJ, Wollan PC, Tefferi A. Population-based incidence and survival figures in essential thrombocythemia and agnogenic myeloid metaplasia: an Olmsted County Study, 1976-1995. Am J Hematol. 1999;61(1):10-15.

5. Tefferi A, Thiele J, Orazi A, Kvasnicka HM, Barbui T, Hanson CA, Barosi G, et al. Proposals and rationale for revision of the World Health Organization diagnostic criteria for polycythemia vera, essential thrombocythemia, and primary myelofibrosis: recommendations from an ad hoc international expert panel. Blood. 2007;110(4):1092-1097.

6. Tefferi A. Myelofibrosis with myeloid metaplasia. N Engl J Med. 2000;342(17):1255-1265.

7. Tefferi A. Pathogenesis of myelofibrosis with myeloid metaplasia. J Clin Oncol. 2005;23(33):8520-8530.

8. Campbell PJ, Griesshammer M, Dohner K, Dohner H, Kusec R, Hasselbalch HC, Larsen TS, et al. V617F mutation in JAK2 is associated with poorer survival in idiopathic myelofibrosis. Blood. 2006;107(5):2098-2100.

9. Kuter DJ, Bain B, Mufti G, Bagg A, Hasserjian RP. Bone marrow fibrosis: pathophysiology and clinical significance of increased bone marrow stromal fibres. $\mathrm{Br} \mathrm{J}$ Haematol. 2007;139(3):351-362.

10. Papageorgiou SG, Castleton A, Bloor A, Kottaridis PD. Allogeneic stem cell transplantation as treatment for myelofibrosis. Bone Marrow Transplant. 2006;38(11):721727.

11. Verstovsek S, Kantarjian H, Mesa RA, Pardanani AD, Cortes-Franco J, Thomas DA, Estrov Z, et al. Safety and efficacy of INCB018424, a JAK1 and JAK2 inhibitor, in myelofibrosis. N Engl J Med. 2010;363(12):11171127.

12. Guardiola P, Anderson JE, Bandini G, Cervantes F, Runde V, Arcese W, Bacigalupo A, et al. Allogeneic stem 
cell transplantation for agnogenic myeloid metaplasia: a European Group for Blood and Marrow Transplantation, Societe Francaise de Greffe de Moelle, Gruppo Italiano per il Trapianto del Midollo Osseo, and Fred Hutchinson Cancer Research Center Collaborative Study. Blood. 1999;93(9):2831-2838.

13. Tefferi A, Barosi G, Mesa RA, Cervantes F, Deeg HJ, Reilly JT, Verstovsek S, et al. International Working Group (IWG) consensus criteria for treatment response in myelofibrosis with myeloid metaplasia, for the IWG for Myelofibrosis Research and Treatment (IWG-MRT). Blood. 2006;108(5):1497-1503.

14. Stewart WA, Pearce R, Kirkland KE, Bloor A, Thomson $\mathrm{K}$, Apperley J, McQuaker G, et al. The role of allogeneic SCT in primary myelofibrosis: a British Society for
Blood and Marrow Transplantation study. Bone Marrow Transplant. 2010;45(11):1587-1593.

15. Deeg HJ, Gooley TA, Flowers ME, Sale GE, Slattery JT, Anasetti C, Chauncey TR, et al. Allogeneic hematopoietic stem cell transplantation for myelofibrosis. Blood. 2003;102(12):3912-3918.

16. Kemp K, Morse R, Wexler S, Cox C, Mallam E, Hows J, Donaldson C. Chemotherapy-induced mesenchymal stem cell damage in patients with hematological malignancy. Ann Hematol. 2010;89(7):701-713.

17. Galotto M, Berisso G, Delfino L, Podesta M, Ottaggio L, Dallorso S, Dufour C, et al. Stromal damage as consequence of high-dose chemo/radiotherapy in bone marrow transplant recipients. Exp Hematol. 1999;27(9):14601466. 